\title{
A Broadband Spectrum Sensing Algorithm in TDCS Based on ICoSaMP Reconstruction
}

\author{
Liu Yang ${ }^{1}$, Ren Qinghua ${ }^{1}$, Xu Bingzheng ${ }^{1}$ and Li Xiazhao ${ }^{1}$ \\ ${ }^{1}$ Information and Navigation College, Air Force Engineering University, Xi'an, China
}

\begin{abstract}
In order to solve the problem that the wideband compressive sensing reconstruction algorithm cannot accurately recover the signal under the condition of blind sparsity in the low SNR environment of the transform domain communication system. This paper use band occupancy rates to estimate sparseness roughly, at the same time, use the residual ratio threshold as iteration termination condition to reduce the influence of the system noise. Therefore, an ICoSaMP(Improved Compressive Sampling Matching Pursuit ) algorithm is proposed. The simulation results show that compared with CoSaMP algorithm, the ICoSaMP algorithm increases the probability of reconstruction under the same SNR environment and the same sparse degree. The mean square error under the blind sparsity is reduced.
\end{abstract}

\section{INTRODUCTION}

Transform domain communication system (TDCS) is one of the candidate technologies of cognitive radio. Because of its good communication and anti-jamming performance in low SNR environment and the advantages of flexible spectrum access mode, it has a good development prospects ${ }^{[1]}$.

TDCS is very suitable for the field of military communication interference due to its strong antijamming performance. However, existing hardware can't bear the pressure of high sampling rate which broadband signals bring, at the same time, in the process of electronic warfare, the interference between hostile parties is usually accurate interference, including single tone interference, multi-tone interference, narrowband interference and Broadband interference, etc. On the other hand, TDCS communication environment is a low signal to noise ratio of the broadband environment. the wireless spectrum has obvious sparse characteristics, so the compressed sensing theory is used into the field of TDCS broadband spectrum sensing.

Reconstruction algorithm is one of the key technologies of compressed sensing. The greedy algorithm in reconstruction algorithm is widely concerned because of its simple structure and high reconstruction probability. The classical reconstruction algorithms include Matching Pursuit algorithm ${ }^{[2]}$, Orthogonal Matching Pursuit ${ }^{[3]}$ and Compressive Sampling Matching Pursuit ${ }^{[4]}$ algorithm. In paper ${ }^{[5]}$, a double threshold orthogonal matching pursuit algorithm is proposed. By double screening the selected atoms, the signal can be reconstructed in the case of blind sparseness, but the maximum number of iterations in the iteration termination condition in the algorithm is not introduced; In order to improve the performance of the reconstructed algorithm based on the noise, a kind of compressed sensing denoising based on selective measurement is proposed in paper [6], However, the algorithm must know the a priori information of the signal sparseness, so the scope of its use is limited. In paper [7], a multi-candidate singular orthogonal matching pursuit algorithm is proposed for the problem that the recovery accuracy of greedy algorithm is not high. However, the algorithm also needs the priori information of sparseness, so the scope of use is limited.

At present, the compressed sensing theory is applied to the field of TDCS broadband spectrum sensing at home and abroad.in paper [8], a MIMO-TDCS compressed-sensing information feedback method based on time-frequency two-dimensional compression is proposed. In paper [9], a method for constructing the deterministic embedded chaotic sequence-cyclic Toeplitz structure compressed sensing observation matrix is proposed for the limitations of random observation matrix in TDCS. these algorithms is based on the premise that the signal sparseness is known. However, the signal sparseness is usually unknown during the actual TDCS transmission.

In view of the above problems, Authors propose an ICoSaMP (Improved Compressive Sampling Matching Pursuit) algorithm. The algorithm uses the frequency occupancy rate to estimate the sparsity of the signal and use the residual ratio threshold as iteration termination condition to reduce the influence of the system noise.

\footnotetext{
* Corresponding author: a2920270@163.com, xubzheng@sina.com
} 
The simulation results show that the ICoSaMP algorithm has a significant improvement in the reconstruction probability compared with the CoSaMP algorithm under the same SNR and sparsity. The mean square error of the ICoSaMP algorithm is lower than that of the CoSaMP algorithm under the blind sparsity.

This section mainly focuses on the background, problems, purpose and significance. Section 2 mainly introduces the model of this paper. Section 3 mainly analyses the CoSaMP algorithm. Section 4 mainly introduces the solution of the problems. Section 5 introduces and analyses the ICoSaMP algorithm this paper proposes. Section 6 mainly simulates the performance of the ICoSaMP algorithm. Section 7 mainly summarizes the full text.

\section{MODEL DESCRIPTION}

Set the working bandwidth of TDCS as B. authorized users randomly occupy part of the band. the rest of band is idle, therefore, the broadband signal has some sparsity in the frequency domain. As shown in Figure 1.

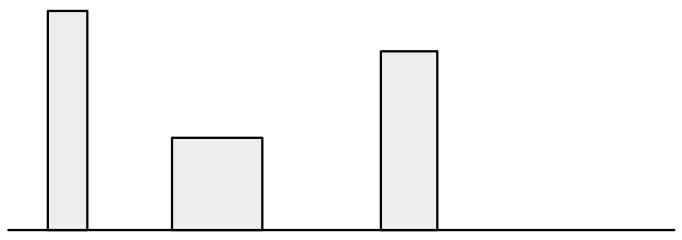

Figure 1: Broadband spectrum sensing model.

In the front of the TDCS, the broadband compressed sensing framework is built, and it is shown in figure 2 .

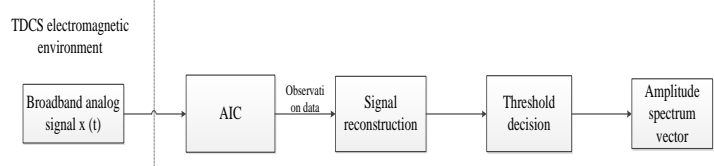

Figure 2: TDCS Broadband Compression sensing Framework.

AIC is an analog information converter, which is based on the compressed sensing theory. It can directly compress signal while sampling, therefore, we can get the observation vector $\mathrm{y}$ is:

$$
\boldsymbol{y}=\boldsymbol{\Phi} \boldsymbol{x}
$$

Where $\boldsymbol{y}$ is the $M \times 1$ dimensional observation vector, and each of the $M$ values contains most of the information of the original signal. Therefore, solving equation (1) belongs to NP problem. In the context of TDCS broadband spectrum sensing, we can take advantage of the sparsity of $\boldsymbol{x}$ in the frequency domain, performing fourier Transform on $\boldsymbol{x}$

$$
\boldsymbol{s}=\boldsymbol{F} \boldsymbol{x}
$$

Where $\boldsymbol{F}$ is an $N \times N$ discrete Fourier transform matrix, $\boldsymbol{s}$ is the spectrum of $\boldsymbol{x}$, having $K$ nonzero values, and $K<M$, It is called the sparseness of the signal $s$. Substituting equation (2) into equation (1)

$$
\boldsymbol{y}=\boldsymbol{\Phi} F^{-1} s
$$

$$
\boldsymbol{y}=\boldsymbol{\Theta s}
$$

Among them, $\boldsymbol{\Theta}$ is an $M \times N$-dimensional observation matrix, which needs to satisfy the conditions such as RIP, Incoherence.

For the solution of Eq. (4), the spectrum $s$ is usually reconstructed by solving a problem based on the minimization of the $e_{0}$ norm, and then the signal $\boldsymbol{x}$ is solved by using equation (2).

$$
\begin{gathered}
\min _{s}\|\boldsymbol{s}\|_{0} \\
\text { s.t. } \quad \boldsymbol{y}=\boldsymbol{\Theta} \boldsymbol{s} \\
\boldsymbol{x}=\boldsymbol{F}^{-1} \boldsymbol{s}
\end{gathered}
$$

The solution obtained by this solution is optimal, but solving equation (5) is still an NP problem. The main method of this method is to transform the $e_{0}$ norm minimization problem into $e_{1}$ norm minimization problem, and use the idea of linear programming to solve the signal reconstruction ${ }^{[10]}$. The paper [11] shows that the minimum norm of $e_{1}$ and $e_{0}$ minimum norm are equivalent under certain conditions. Thus equation (5) can be written as

$$
\begin{aligned}
& \underset{s}{\min }\|\boldsymbol{s}\|_{1} \\
& \text { s.t. } \boldsymbol{y}=\boldsymbol{\Theta} \boldsymbol{s}
\end{aligned}
$$

At present, this paper mainly uses the greedy algorithm as a fast and effective algorithm to solve the above-mentioned reconstruction problem. At present, it mainly includes matching pursuit algorithm (MP), stagewise orthogonal matching pursuit algorithm (StOMP), orthogonal matching pursuit algorithm (OMP) and compressive sampling matching pursuit algorithm (CoSaMP) and so on.

\section{COSAMP ALGORITHM}

Compared with other greedy algorithms, the advantage of CoSaMP algorithm is that it can identify multiple atoms in each iteration process, and can converge quickly. At the same time, we add the idea of "back check", which improves the reconstruction precision and avoids the threshold selection problem. The algorithm pseudo code is as follows.

Input: observation matrix $\boldsymbol{\Theta}$, measured value $\boldsymbol{y}$, sparse degree $K$.

Output: rebuild signal $\hat{\boldsymbol{x}}$.

Initialize: $\hat{\boldsymbol{x}}_{0}=0$, residual $\boldsymbol{r}_{0}=\boldsymbol{y}$, iterations identify $m=0$, and the index $\Lambda_{0}$ set is empty.

(1) $m=m+1$.

(2) generate the intermediate agent signal $\boldsymbol{u}=\boldsymbol{\Theta}^{T}{ }_{m-1} \boldsymbol{r}_{m-1}$, find $\boldsymbol{u}$ in the $2 K$ large component of the location set $\Omega$.

(3)update $\Lambda_{m}=\Lambda_{m-1} \cup \Omega$ and update the column collection $\boldsymbol{\Theta}_{m}=\left[\begin{array}{ll}\boldsymbol{\Theta}_{m-1} & \boldsymbol{\Theta}_{\Omega}\end{array}\right]$.

(4) reconstruct the signal, through the least squares estimation, get $\tilde{\boldsymbol{x}}=\boldsymbol{\Theta}_{m}^{\dagger} \boldsymbol{y}$ and retain the $\mathrm{K}$ maximum component, then get $\hat{\boldsymbol{x}}$.

(5) update the residual, $\boldsymbol{r}_{m}=\boldsymbol{y}-\boldsymbol{\Theta} \hat{\boldsymbol{x}}$. 
(6) terminate the decision, if $m<K$, then return (1), otherwise (7).

(7) output $\hat{\boldsymbol{x}}$.

In the above equation, $\boldsymbol{\Theta}^{\dagger}$ is called $\boldsymbol{\Theta}$ 's pseudo inverse. Although CoSaMP algorithm has the advantages of fast convergence and good anti-noise performance, its biggest flaw is that it needs to know the a priori information of signal sparseness, so its practical application potential is very limited.

\section{ITERATION TERMINATION CONDITION OF RESIDUAL RATIO THRESHOLD}

In order to solve the shortcoming of the traditional iterative termination condition, a new iterative termination condition is proposed by the paper [12], which solves the shortcoming of the traditional iteration termination condition under low SNR.

First, the measured value y can be decomposed into

$$
\boldsymbol{y}=\hat{\boldsymbol{y}}+\widehat{\boldsymbol{e}}_{B}+\boldsymbol{e}_{B}
$$

Among them, $\hat{y}$ is the noise-free interference signal. $\widehat{\boldsymbol{e}}_{B}$ is the noise for the bandwidth $B$. $\boldsymbol{e}_{B}$ is the outside the noise for the bandwidth $B . \boldsymbol{e}_{B}$ and $\hat{\boldsymbol{y}}$ are independent of each other, then the mth iteration $\left\|\boldsymbol{r}_{m}(y)\right\|_{2}^{2}$ can be written in the following form

$$
\begin{aligned}
& \left\|\boldsymbol{r}_{m}(y)\right\|_{2}^{2}=\left\|\boldsymbol{r}_{m}\left(\hat{y}+\widehat{e}_{B}+e_{B}\right)\right\|_{2}^{2} \\
& \quad\left\|\boldsymbol{r}_{m}(y)\right\|_{2}^{2}=\left\|\boldsymbol{r}_{m}\left(\hat{y}+\widehat{e}_{B}\right)\right\|_{2}^{2}+\left\|\boldsymbol{e}_{B}\right\|_{2}^{2}
\end{aligned}
$$

Similarly, $\left\|\boldsymbol{r}_{m+1}(y)\right\|_{2}^{2}$ is

$$
\left\|\boldsymbol{r}_{m+1}(y)\right\|_{2}^{2}=\left\|\boldsymbol{r}_{m+1}\left(\hat{y}+\widehat{e}_{B}\right)\right\|_{2}^{2}+\left\|\boldsymbol{e}_{B}\right\|_{2}^{2}
$$

With the increase in the number of iterations, the change of the $\left\|\boldsymbol{r}_{m}\left(\hat{y}+\widehat{e}_{B}\right)\right\|_{2}^{2}$ will be smaller and smaller, which is not enough to change $\left\|\boldsymbol{r}_{m}(y)\right\|_{2}^{2}$, then $\left\|\boldsymbol{e}_{B}\right\|_{2}^{2}$ become the main factor of the impact in $\left\|\boldsymbol{r}_{m}(y)\right\|_{2}^{2}$. Accordingly, the difference between the residuals of the two iterations is more likely to reflect the iteration, and thus the iterative termination condition is

$$
f_{m}=\frac{\left\|\boldsymbol{r}_{m}-\lambda_{m} \boldsymbol{r}_{m-1}\right\|_{2}^{2}}{\left\|\lambda_{m} \boldsymbol{r}_{m-1}\right\|_{2}^{2}}<\theta
$$

In this equation, $\lambda_{m}=\sqrt{\frac{\left\|\boldsymbol{r}_{m}\right\|_{2}^{2}}{\left\|\boldsymbol{r}_{m-1}\right\|_{2}^{2}}}$ is the residual coefficient, which can reduce the influence of random noise and enhance the robustness of the algorithm. $\theta$ is the set threshold and is independent of channel characteristics and noise.

In this paper, the CoSaMP algorithm based on the above ideas is improved, and an ICoSaMP algorithm is proposed.

\section{ICOSAMP ALGORITHM}

\subsection{ALGORITHM ANALYSIS}

According to the analysis of CoSaMP algorithm in Section 3, its biggest drawback is that we need to know the prior information such as signal sparseness and use it as the number of iterations, which greatly reduces its potential in practical application. In the fourth section, for the shortcoming of the traditional iteration termination condition, a residual ratio threshold iteration termination condition is proposed, which overcomes the influence of the process of reconstructing the signal at lower SNR.

From the above analysis, the residual ratio threshold iteration termination condition can be applied to the CoSaMP algorithm as an iteration termination condition without relying on the signal sparse degree $K$. that is, the iteration can be calculated once for each time the residual ratio $f_{m}$, and then with the set threshold $\theta$ is compared, if $f_{m}<\theta$, the iteration stops and outputs $\hat{\boldsymbol{x}}$, otherwise iterates until $f_{m}<\theta$. For the CoSaMP algorithm in the iterative process of the sparse degree $K$, can be estimated by the occupation of the band.

In TDCS practical engineering applications, the $K$ of the signal is difficult to obtain, but the occupancy rate of a band is very easy to obtain, usually through the spectrum detection equipment in a certain area to detect a band, or through The relevant spectrum management department to obtain long-term survey results. after getting the band occupancy rate, the signal can be a slight estimate of the degree of sparseness. for example, the relevant US research departments on the $30 \mathrm{MHz}$ $3 \mathrm{GHz}$ frequency band within the spectrum of occupation , The result is $5.2 \% \sim 13.1 \%$, so the range of sparseness of this band is $N \times 5.2 \% \sim N \times 13.1 \%$, where $N$ is the Nyquist sampling frequency and the sparsity estimate $K$ 'can be taken in this range.

\subsection{ALGORITHM FLOW}

The code of the ICoSaMP algorithm is as follows.

Input observation matrix $\boldsymbol{\Theta}$, observed value $\boldsymbol{y}$, estimation of sparsity $K^{\prime}$.

(1) initialization: $\hat{\boldsymbol{x}}=0$, residual $\boldsymbol{r}_{0}=\boldsymbol{y}$, the number of iterations identified $m=0$, the index $\Lambda_{0}$ set is empty.

(2) $m=m+1$.

(3) generate the intermediate agent signal $\boldsymbol{u}=\boldsymbol{\Theta}^{T}{ }_{m-1} \boldsymbol{r}_{m-1}$, find $\boldsymbol{u}$ in the $2 K^{\prime}$ large component of the location of the collection $\Omega$.

(4) update $\Lambda_{m}=\Lambda_{m-1} \cup \Omega$ and update the column collection $\boldsymbol{\Theta}_{m}=\left[\begin{array}{ll}\boldsymbol{\Theta}_{\mathrm{m}-1} & \boldsymbol{\Theta}_{\Omega}\end{array}\right]$.

(5) reconstruct the signal, by least squares estimation, getting $\tilde{\boldsymbol{x}}=\boldsymbol{\Theta}_{m}^{\dagger} \boldsymbol{y}$ and retain the $K^{\prime}$ largest components. Then get $\hat{\boldsymbol{x}}$.

(6) update the residual, $\boldsymbol{r}_{m}=\boldsymbol{y}-\boldsymbol{\Theta} \hat{\boldsymbol{x}}$. 
(7) iterative decision, if $f_{m}<\theta$, implement (8), otherwise return (2), the threshold value $\theta$ range is usually 0.1 to 0.5 .

(8) output $\hat{\boldsymbol{x}}$.

The algorithm ends.

In the improved ICoSaMP algorithm, do not need to know the signal sparse degree $K$, which is a priori information. we just need to know the frequency band of the study instead, and this parameter in the actual application process is easy to obtain. In addition, another advantage of the algorithm is the fusion of residuals ratio threshold iteration termination conditions and then the step (7) iterative decision process, instead of the signal sparsity of this iteration termination condition. Which solves the problem that CoSaMP algorithm can not reconstruct the signal accurately under TDCS blindness.

\section{SIMULATION ANALYSIS}

\section{1 ANALYSIS}

In order to verify the reconstruction effect of the ICoSaMP algorithm, especially the difference in the reconstruction probability between ICoSaMP algorithm and other algorithms in the lower SNR of TDCS. We use MATLAB simulation platform for verification. select Gaussian random Measurement matrix as measurement matrix. Nyquist sampling frequency points $N=256$, the number of observations $M=128$, band occupancy rate $\eta$ $=10 \%$, decision threshold $\theta=0.2$.

\subsubsection{SIMULATION ANALYSIS OF RECONSTRUCTION PROBABILITIES WITHOUT NOISY CONDITIONS}

Figure 3 shows the comparison of the reconstruction effects of OMP, ROMP, StOMP, CoSaMP and ICoSaMP algorithms without noisy conditions.

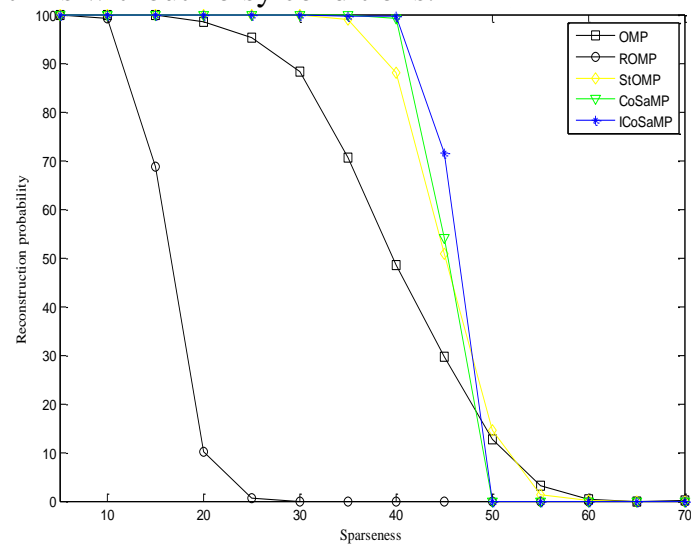

Figure 3: Comparison of reconstruction probabilities of different algorithms without noisy conditions.

It can be seen from the figure that the reconstruction probability curve of ICoSaMP algorithm is better than that of the previous CoSaMP algorithm. When the signal sparseness is lower than 13, each algorithm can reconstruct the original signal with the probability of nearly $100 \%$. When the signal sparseness is between 15 and 40, The reconstruction probability of OMP and ROMP began to decrease obviously, while CoSaMP and ICoSaMP algorithm can reconstruct the original signal with the probability of nearly $100 \%$. When the sparse degree is greater than 45 , the reconstruction probability of CoSaMP and ICoSaMP algorithm begins to drop even lower than that of the previous Several algorithms. This may be due to CoSaMP and ICoSaMP algorithm need to select more atoms as a support set and resulting in over match in the higher sparse degree. so in the lower sparse degree, the performance of ICoSaMP algorithm reconstruction is obvious.

\subsubsection{SIMULATION ANALYSIS OF RECONSTRUCTION PROBABILITIES UNDER NOISY CONDITIONS.}

The working environment of TDCS transmission system is the low SNR environment, therefore, it is more significant to compare the performances of each algorithms' reconstruction probability in the low SNR environment. The experimental results are shown in the following figure.

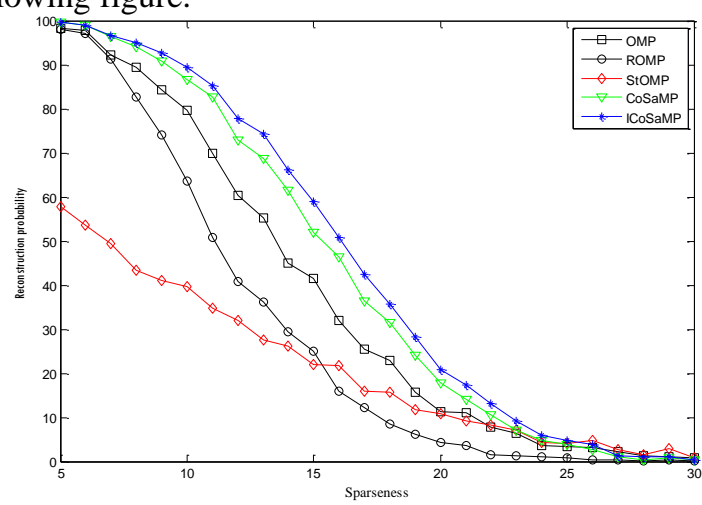

Figure 4: Comparison of reconstruction probabilities for different algorithms at $\mathrm{SNR}=1$

Figure 4 shows the relationship between the reconstruction probability and the sparseness of the algorithm under the low SNR. It can be seen from the figure that the reconstruction probability of each algorithm is affected by different degree in the environment of lower SNR. The reconstruction probability of ICoSaMP algorithm is better than that of other algorithms. The calculation probability of ICoSaMP algorithm is about $10 \%$ higher than that of CoSaMP algorithm in the same SNR environment. This is because ICoSaMP algorithm uses the difference between adjacent iterations and the residual ratio coefficient to weaken the influence of random noise, and then improve its anti-noise performance. Therefore, the advantages of ICoSaMP algorithm in the lower SNR environment is more obvious. 


\subsection{MEAN SQUARE ERROR (MSE) ANALYSIS}

In order to test the recovery accuracy of ICoSaMP algorithm in TDCS low signal-to-noise ratio (SNR), this paper use mean square error (MSE) simulation analysis, signal sparseness $K=25, \quad$ spectrum occupancy rate $\eta=10 \%$, the measurement matrix selects the Gaussian random measurement matrix, Nyquist sampling frequency points $N=256$, the number of observations $M=128$, the decision threshold $\theta=0.2$. The simulation results are shown in the following figure.

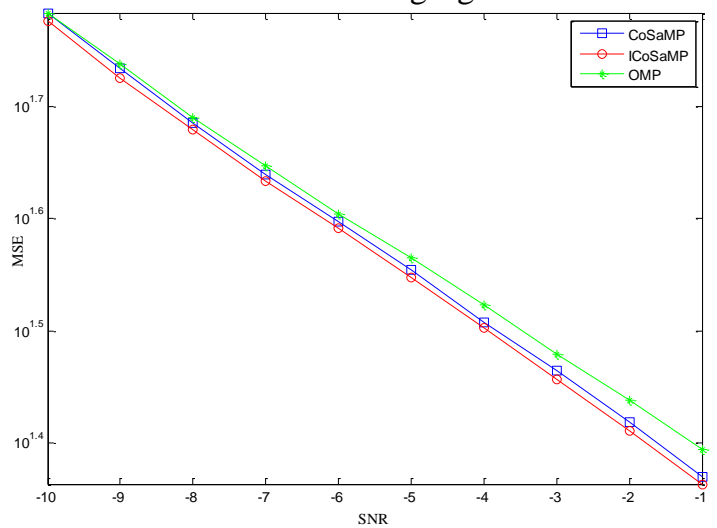

Figure 5: Comparison of signal recovery MSE for different algorithms

Figure 5 shows the curve of MSE value with the signal to noise ratio. Because TDCS still has good performance at lower SNR, the simulation environment in this paper is lower SNR. It can be seen from the figure that the MSE is reduced with the increase of the SNR, and the MSE of the ICoSaMP algorithm is slightly better than the CoSaMP algorithm. This is due to the ICoSaMP algorithm treats the residuals of the adjacent two iterations as the iteration termination condition and increases the residual ratio coefficient. These improved measures make the ICoSaMP algorithm have some antinoise performance, so the accuracy of the recovered signal is higher than that of the CoSaMP algorithm in the TDCS lower SNR environment.

\section{CONCLUSIONS}

In the TDCS low SNR environment, the signal in the frequency domain has obvious sparse characteristics, so use the compressed sensing technology to sense TDCS front spectrum environment. In practical engineering applications, the sparsity of the signal is difficult to obtain, and the traditional CoSaMP algorithm in the use of the signal reconstruction needs to know this priori information. In this paper, the sparse degree of the signal is estimated by spectrum occupancy situation, and the CoSaMP algorithm is improved by using the residual ratio threshold as an iteration termination condition, so the ICoSaMP algorithm is proposed. The simulation results show that ICoSaMP algorithm is more accurate than CoSaMP algorithm, and its anti-noise performance is stronger, which is significant for wideband spectrum sensing in TDCS low SNR.

\section{REFERENCES}

1. T. C. XIE, X. Y. Da and Z. Y. Chu, "An estimation algorithm of basis function synchronous paramenters of Transform Domain Communication Systems based on Frobenius Norm" Journal of Air Force Engineering University, vol. 15(1), Feb. 2014, pp. 57-61.

2. S. G. Mallat and Z. Zhang, "Matching pursuits with timefrequency dictionaries" IEEE Transactions on Signal Processing, vol. 41(12), 1993, pp. 3397 - 3415.

3. J. A. Tropp and A. C. Gilbert, "Signal Recovery From Random Measurements Via Orthogonal Matching Pursuit" IEEE Transactions on Information Theory, vol. 53(12), 2007, pp. 4655-4666.

4. D. Needell and J. A. Tropp, "CoSaMP: Iterative Signal Recovery From Incomplete and Inaccurate Samples" Applied \& Computational Harmonic Analysis, vol. 26(3), 2009, pp. 301-321.

5. X. Y. Liu, Z. G. Zhao and H. X. Lv, "Double threshold Orthogonal Matching Pursuit algorithm" Computer Science, vol. 44, June. 2017, pp. 212-215.

6. L. Y. Pei, H. Jiang and R. L. Ma, "Denoising recovery for compressive sensingbased on selective measure," Journal on Communications, vol. 38, February. 2017, pp. 106-114

7. J. P. Tian, X. J. Liu and Y. P. Liu, "Multi-candidate Set of Generalized Orthogonal Matching Pursuit Algorithm" Journal of Applied Sciences, vol. 35(2), 2017, pp. 233-243.

8. C. L. Gao and Q. H. Ren, "Study on MIMO-TDCS timefrequency compressed sensing feedback scheme" Semiconductor Optoelectronics, vol. 35 june. 2014, pp. 492-496.

9. N. Li, Q. H. Ren and Y. Z. Su. "Constructive method of CS measurement matrix in TDCS" Computer Engineering \& Design, vol. 38, Jan. 2017, pp. 7-11.

10. Q. Li and X. Zhao, "Adaptive OMP Algorithm for Wideband Spectrum Sensing" Journal of Jilin University, vol. 33, Mar. 2015, pp. 113-119.

A. M. MADNI, "A systems perspective on compressed sensing and its use in reconstructing sparse networks" IEEE Systems Journal, vol. 8(1), 2014, pp. 23-27.

11. F. H. Fan and H. L. Ruan, "Non-convex compressive sensing ultra-wide band channel estimation method in low SNR conditions" Acta Electronica Sinica, vol. 42(2), Feb. 2014, pp. 353-359.

12. Y. Chen and Z. QIN, "Gradient-based compressive image fusion" Frontiers of Information Technology \& Electronic Engineering, vol.16(3), 2015, pp. 227-237.

13. J. S. Dong, J. Y. Yin and C. F. LI, "A gradient-based steering kernel reconstruction strategy for semi-random Fourier measurements in compressed remote sensing" Journal of Infrared \& Millimeter Waves, vol. 34(6), 2015, pp. 673-679.

14. J. Y. Zhuang, C. Qian and W. J. He, "Imaging through dynamic scattering media with compressed sensing" Acta Physica Sinica, vol. 65(4), 2016, pp. 22-31. 